\title{
Whole Exome Sequencing Revealed a Candidate Gene for Finkelstein-Seidlmayer Disease
}

\author{
Gabriel Bronz ${ }^{1, ~ *, ~ H e i n z ~ G a b r i e l ~}{ }^{2}$, Sebastiano Antonio Lava ${ }^{3}$, Gian Paolo Ramelli ${ }^{4,5}$, \\ Manuel Luedeke ${ }^{2}$, Saskia Biskup ${ }^{2}$, Carlo Mainetti ${ }^{6}$, Alessandra Ferrarini ${ }^{7,8}$ \\ ${ }^{1}$ Medical Faculty, University of Bern, Bern, Switzerland \\ ${ }^{2}$ Centre for Genomics and Transcriptomics, Tuebingen, Germany \\ ${ }^{3}$ Pediatric Cardiology Unit, Department of Pediatrics, Centre Hospitalier Universitaire Vaudois (CHUV), Lausanne, Switzerland \\ ${ }^{4}$ Service of Neuropediatry, Hospital of Bellinzona, Bellinzona, Switzerland \\ ${ }^{5}$ Medical Faculty, University of Basel, Basel, Switzerland \\ ${ }^{6}$ Service of Dermatology, Hospital of Bellinzona, Bellinzona, Switzerland \\ ${ }^{7}$ Service of Medical Genetics, Italian Hospital of Lugano, Lugano, Switzerland \\ ${ }^{8}$ Medical Faculty, University of Lugano, Lugano, Switzerland
}

\section{Email address:}

gabriel.bronz@students.unibe.ch(G. Bronz),Heinz.Gabriel@humangenetik-tuebingen.de(H. Gabriel),

webmaster@sebastianolava.ch(S. A. Lava), Gianpaolo.Ramelli@eoc.ch (G. P. Ramelli),

Manuel.Luedeke@humangenetik.tuebingen.de(M. Luedeke), Saskia.Biskup@humangenetik-tuebingen.de(S. Biskup),

Carlo.Mainetti@eoc.ch (C.Mainetti),Alessandra.Ferrarini@eoc.ch(A. Ferrarini)

${ }^{*}$ Corresponding author

\section{To cite this article:}

Gabriel Bronz, Heinz Gabriel, Sebastiano Antonio Lava, Gian Paolo Ramelli, Manuel Luedeke, Saskia Biskup, Carlo Mainetti, Alessandra Ferrarini. Whole Exome Sequencing Revealed a Candidate Gene for Finkelstein-Seidlmayer Disease. American Journal of Pediatrics. Vol. 5, No. 4, 2019, pp. 196-199. doi: 10.11648/j.ajp.20190504.15

Received: August 6, 2019; Accepted: September 11, 2019; Published: September 26, 2019

\begin{abstract}
Background: Finkelstein-Seidlmayer disease (FSD) is a benign cutaneous small-vessel leukocytoclastic vasculitis syndrome, which normally affects children between 2-60 months in a male-to-female ratio of 2:1. Skin lesions may appear as papules, erythematous macules, or urticaria. They are symmetric, sharp-edged and favouring the face, ears and extremities. Frequently they are targetoid, annular, medallion-like, or cockade. Fever and extracutaneous involvement are rare and spontaneous resolution occurs in 1-3 weeks. Case Information: In 2015, we reported a familial occurrence of FSD. Patients described in that article were the mother and all her three sons. All of them had a history of recurrent and relapsing nonthrombocytopenic, red-to-purpuric skin lesions, with a neonatal-onset. There was no systemic involvement. Anamnestic data revealed that maternal aunt, cousin and grandmother had also a positive history of neonatal onset of an acute cockade purpura and oedema. At that time, we suspected a genetic form of FSD with an autosomal dominant transmission or X-linked inheritance and incomplete penetrance. Method and Results: Blood samples were obtained from all available family members and a whole exome sequencing (WES) was performed on various affected and non-affected members of this family. The genetic analysis identified a common new mutation in the HCK gene. Conclusion: Up now, FSD is considered a sporadic disease and no genetic researches have been published on affected patients. We performed WES on a previously reported familiar case of FSD and the result was a common mutation in $H C K$ gene. We found out the mutation on all the analysed affected and obligate-carrier members of the family. $H C K$ gene encodes for a hematopoietic cell kinase protein, which is a member of the SRC family of cytoplasmic tyrosine kinases $(S F K)$. We propose that $H C K$ gene could be a candidate gene in the pathophysiology of some types of FSD. We also discuss the autosomal dominant transmission and incomplete penetrance of these specific types of disease.
\end{abstract}

Keywords: Finkelstein-Seidlmayer Disease, Acute Cockade Purpura, Oedema of Young Children, Small-vessel Leukocytoclastic Vasculitis, Whole Exome Sequencing 


\section{Introduction}

Finkelstein-Seidlmayer disease (FSD), also known as acute cockade purpura and oedema of young children, is a rare and benign cutaneous small-vessel leukocytoclastic vasculitis syndrome. The patients present non-pruriginous target-like lesions, frequently associated with tender, nonpitting edema. The lesions affect mostly the extremities, ears and face. Normally, the affected children are between 2-60 months and in a male-to-female ratio of $2: 1$. This disease usually follows a febrile illness or an active immunization and it typically does not recur [1].

In 2015, we reported a familial occurrence of FSD [2]. Patients reported in that article were the 46-year-old mother and her three affected sons, aged 14, 12 and 9. All of them had a history of recurrent and relapsing nonthrombocytopenic, red-to-purpuric skin lesions, with a neonatal-onset. There was no systemic involvement. Other relatives in the family developed an acute cockade purpura and oedema (Figure 2; II-D; III-E; IV-G), but their genetic material was not available.

Subsequent reconstruction of family history revealed that the paternal grandmother of the patient had also presented a clinical history of FSD.

At that time, we supposed autosomal dominant transmission or, less likely, X-linked inheritance, with a possible incomplete penetrance.

By whole exome sequencing (WES) we identified a candidate gene for FSD, supporting our hypothesis of an autosomal dominant and possibly monogenic transmission of the disease.

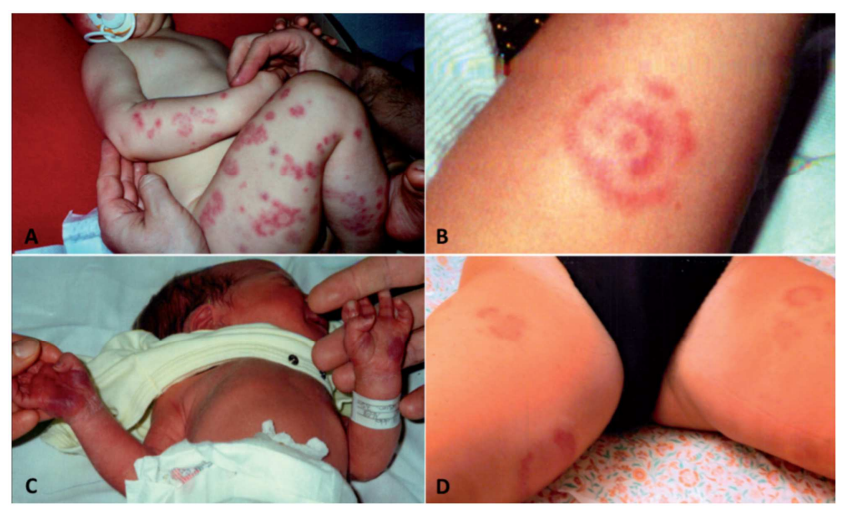

Figure 1. pictures of our patients diagnosed with FSD reported in 2015. Pictures from [2] (A) Haemorrhagic lesions on the extremities of son 1 at the age of 3 months. (B) Target-like lesion on the leg of son 1 at 15 months of age. (C) Hemorrhagic plaques at birth on the palmar side of the hands of son 1. (D) Annular oedematous thigh plaques in the mother at 34 years of age.

\section{Methods and Results}

Blood samples were obtained from all available family members (III-C; III-D; IV-D; V-A; V-B; V-C) and the DNA was extracted accordingly by standard procedures; written consent was obtained. The coding and flanking regions were enriched using the Agilent in solution technology and were sequenced using the Illumina Hiseq/NovaSeq/system. At least one causative or rare variant was re-sequenced using conventional Sanger sequencing, providing a second, independent confirmation of the used DNA sample. Only variants in the coding region and the flanking intronic regions (+/-8bp) with a minor allele frequency (MAF) $(<1.5 \%)$ were considered. Known disease - causing variants were evaluated in up to $+/-30 \mathrm{bp}$ of flanking region and up to $5 \%$ MAF.

Minor allele frequencies were taken from public database (ex. GnomAD) and an in-house database. Candidate CNV were evaluated manually. Potentially pathogenic variants were confirmed with a second method (ex. MLPA).

Patient (IV-D), her three affected sons (V-A; V-B; V-C) and her father (III-D) shared the same variant c. $1555 \mathrm{G}>\mathrm{T}$; $\mathrm{p}$. Glu519* in the HCK gene in a heterozygous state. The variant was not present in the mother of the patient (III-C) (Figure 2).

\section{Discussion}

FSD is a cutaneous leukocytoclastic small-vessels vasculitis, with an impressive clinical picture and presenting purpuric, non-painful large skin plaques and oedema. Normally, the patient's general condition is good, visceral involvement is very uncommon. It is considered a minor form of Henoch-Schoenlein syndrome (HSP).

Various infectious agents has been identified as trigger for a FSD; among them Mycoplasma Pneumoniae, Streptococcus Pneumoniae, Hepatitis A virus, Cytomegalovirus, Staphylococcus, Campylobacter, Coxsackie virus, Adenovirus and Rotavirus. Some patients reported a temporal association with vaccines injections. Up now, no genes are associated with the FSD [1-6].

Whole exome sequencing permitted us to identify a candidate gene, which might be responsible for the FSD in a family we previously reported [2].

The heterozygous variant c. $1555 \mathrm{G}>\mathrm{T}$; p. Glu519* in the $H C K$ gene was identified first in the patient (IV-D) and one of her affected sons (V-A). Segregation analysis was subsequently performed for the two other affected sons (V-B and V-C). Finally - as the familial history indicated a paternal transmission of the disease - the mother and the father (III-C and III-D) of the patient (IV-D) were analysed and the same $H C K$ variant was detected in the father (III-D) (Figure 2).

In all the patients with clinical manifestations of FSD (IV$\mathrm{D}$; V-A; V-B; V-C) and the patient without manifestations, but hypothesized to be a carrier (III-D), the c. $1555 \mathrm{G}>\mathrm{T}$; p. Glu519* variant in $H C K$ was present in a heterozygous state.

The variant reported in our patients resulted in a premature stop codon within the last exon of $H C K$, which will most likely lead to a truncated protein and a loss of the 8 aminoacids at the $\mathrm{C}$-terminal end. The variant has not been reported before in the population dataset gnomAD or in the clinical- 
genetic database ClinVar. According to the current state of any disease in humans. knowledge, variants in the $H C K$ gene are not known to cause

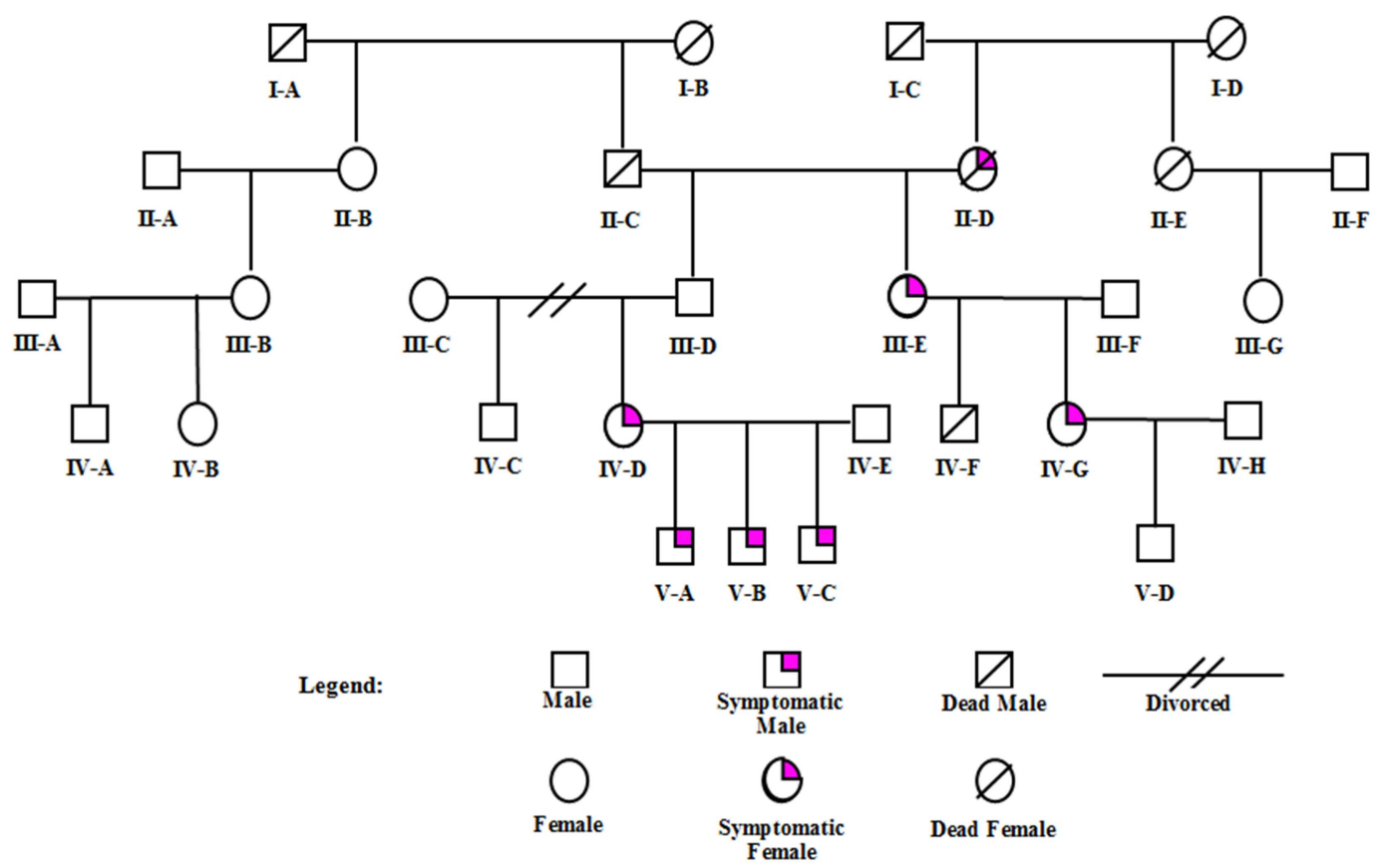

Figure 2. Genealogical tree of our cases reported in one family. The symptomatic patients are indicated with a pink square. In the patients III-C; III-D; IV-D; $V-A ; V-B ; V-C$ a Whole Exome Sequencing was performed. The genetic material of the other symptomatic patients (II-D; III-E; IV-G) was not available.

Gene function and type of mutation strongly suggest that $H C K$ is linked in our case family to the FSD. Our data also suggests that the variant in $H C K$ is transmitted as an autosomal dominant trait with - probably - incomplete penetrance; hypothesis confirmed by the presence of the mutation in the asymptomatic but obligate carrier. These findings warrant further analysis of additional sporadic and familial FSD patients, in order to confirm our hypothesis.

\section{Conclusion}

New technologies permit to discover genetic bases and predisposition to rare and common diseases.

In 2015 we described a family where various members had a history of FSD suggesting a possible strong role of one or more genes.

In the era of WES, this technology permitted us to identify a common truncating variant in the $H C K$ gene. $H C K$ gene encodes the hematopoietic cell kinase protein, which is a member of the SRC family of cytoplasmic tyrosine kinases $(S F K)$. SFKs have a similar protein domain organization, consisting of a unique domain at the N-terminal end, the Src homology 3 and 2 domains (SH3 and $\mathrm{SH} 2$ ), a domain with kinase activity and a C-terminal tail including a phosphorylation site for the cytoplasmic tyrosine kinase $(C S K)$ [7-9].

The kinase $H C K$ is predominantly expressed in granulocytic and monocytic cells, and B-lymphocytes. It is involved in various cellular processes of the immune system. It may play a role in the degranulation of neutrophils. Aberrant activity has been reported in inflammation and cancer [9].

While no genetic base has been suggested before for the FSD, recent studies evidenced a possible association between some genetic polymorphisms and the HSP. These polymorphisms are for example located in genes coding renin-angiotensin-system (RAS) components, endothelial nitric oxide synthases, Inter-Cellular Adesion Molecule 1, and Vascular Endotelial Growth Factor [10-14]. Also, MEFV, the gene responsible for the Familial Mediterranean Fever, could be a candidate gene for HSP [15-17]. If we consider FSD and HSP variants of a same clinical spectrum disease, our finding support the hypothesis that genes involved in inflammatory/immunological processes could cause directly or in association with a trigger (infection?) both FSD and/or HSP. In the near future, the availability of new technologies will answer to old questions laying the groundwork for gene therapies.

\section{References}

[1] Fiore E, Rizzi M, Simonetti GD, Garzoni L, Bianchetti MG, Bettinelli A. 2011. Acute hemorrhagic edema of young children: a concise narrative review. Eur J Pediatr. 170: 15071511 .

[2] Ostini A, Ramelli GP, Mainetti C, Bianchetti MG, Ferrarini A. 2015. Recurrent Finkelstein-Seidlmayer Disease in Four Firstdegree Relatives. Acta Derm Venereol. 95: 622-623.

[3] Miconi F, Cassiani L, Savarese E, Celi F, Papini M, Principi N, Esposito S. 2019. Targetoid skin lesions in a child: acute hemorragic oedema of infancy and its differential diagnosis. Int J Environ Res Public Health. 16 (5): 823. 
[4] Ferrarini A, Milani GP, Bianchetti MG, Lava SAG. 2018. Acute hemorragic edema of infancy associated with coxsackie virus infection. Arch Pediatr. 25: 244.

[5] Savino F, Lupica MM, Tarasco V, Locatelli E, Viola S, di Montezemolo LC, Coppo P. 2013. Acute hemorrhagic edema of infancy: A troubling cutaneous presentation with a selflimiting course. Pediatr. Dermatol. 30: 149-152.

[6] Ferrarini A, Milani GP, Bianchetti MG, Lava SAG. 2018. Acute hemorrhagic edema of infancy associated with Coxsackie virus infection. Arch. Pediatr. 25: 244.

[7] Okada M. 2012. Regulation of the SRC family kinases by Csk. Int J Biol Sci. 8 (10): 1385-1397.

[8] Mitchell J, Kim SJ, Seelmann A, Veit B, Shepard B, Im E, Rhee SH. 2018. Src family kinase tyrosine phosphorylates Toll-like receptor 4, to dissociate MyD88 and al/Tirap, suppressing LPS-induced inflammatory responses. Biochem Farmacol. 147: 119-127.

[9] Roseweir AK, Powell AGMT, Horstman SL, Inthagard J, Park JH, McMillan DC, Horgan PG, Edwards J. 2019. Src family kinases, HCK and FGR, associate with local inflammation and tumour progression in colorectal cancer. Cell Signal. 56: 1522.

[10] Ozkaya O, Söylemezoğlu O, Gönen S, Misirlioğlu M, Tuncer S, Kalman S, Buyan N, Hasanoğlu E. 2006. Renin-angiotensin system gene polymorphisms: association with susceptibility to Henoch-Schonlein purpura and renal involvement. Clin Rheumatol 25 (6): 861-5.

[11] Desong L, Fang L, Songhui Z, Liu W, Shi M, Xiuying C,
Liqun D, Yannan G, Jin W, Zheng W. 2010. Renin-angiotensin system gene polymorphisms in children with HenochSchönlein purpura in West China. J Renin Angiotensin Aldosterone Syst11 (4): 248-55.

[12] Wang A, Wang A, Xiao Y, Wang J, Xu E. 2017. Association of Endothelial Nitric Oxide Synthase Gene Polymorphism with Susceptibility and Nephritis Development of HenochSchönlein Purpura in Chinese Han Children. Genet Test Mol Biomarkers 21 (6): 373-381.

[13] Zhong W, Zhou TB, Jiang Z. 2015. Association of endothelial nitric oxide synthase gene polymorphism with the risk of Henoch-Schönlein purpura/Henoch-Schönlein purpura nephritis. Ren Fail 37 (3): 372-6.

[14] Topaloglu R, Sungur A, Baskin E, Besbas N, Saatci U, Bakkaloglu A. 2001. Vascular endothelial growth factor in Henoch-Schonlein purpura. J Rheumatol 28 (10): 2269-73.

[15] He X, Yu C, Zhao P, Ding Y, Liang X, Zhao Y, Yue X, Wu Y, Yiin W. 2013. The genetics of Henoch-Schoenlein Purpura: a sistematic reviews and meta-analysis. Rheumatol Int 33 (6): $1387-1395$.

[16] Ekinci RMK, Balci S, Bisgin A, Atmis B, Dogruel D, Altintas DU, Yilmaz M. 2019. MEFV gene variants in children with Henoch-Schönlein purpura and association with clinical manifestations: a single-center Mediterranean experience. Postgrad Med 131 (1): 68-72.

[17] Altug U, Ensari C, Sayin DB, Ensari A. 2013. MEFV gene mutations in Henoch-Schönlein purpura. Int J Rheum Dis 16 (3): $347-51$ 